\title{
Critical Complements: Progress on the Orbit of T Mon ${ }^{1}$
}

\author{
N. R. Evans
}

Harvard Smithsonian Center for Astrophysics, Cambridge, MA 02138, USA

K. Carpenter

LASP, NASA/Goddard Space Flight Center, Greenbelt, MD 20771, USA

R. Robinson

CSC, NASA/Goddard Space Flight Center, Greenbelt, MD 20771, USA

D. Massa

Raytheon STX Cor, NASA/Goddard Space Flight Center, Greenbelt, MD 20771, USA

G. M. Wahlgren

Dept. of Physics, University of Lund, S-22362 Lund, Sweden

J. Vinkó

Dept. of Optics, JATE University, H-6720 Szeged, Hungary

L. Szabados

Konkoly Observatory, P.O. Box 67, H-1525 Budapest XII, Hungary

Abstract. A preliminary orbit has been derived for the high luminosity, 27-d classical Cepheid T Mon. Velocities for the hot companion have been measured from an HST GHRS spectrum and 3 IUE spectra. The companion velocities are inconsistent with binary orbital motion and it is likely that the companion is itself a short period binary. The HST spectrum also shows that the companion is a chemically peculiar star, probably magnetic.

\footnotetext{
${ }^{1}$ Based on observations made with the NASA/ESA Hubble Space Telescope, obtained at the Space Telescope Science Institute, which is operated by the Association of Universities for Research in Astronomy, Inc. under NASA Contract No. NASA-26555
} 


\section{Introduction}

Galactic Cepheids can provide some "critical complements", parameters needed for a quantitative interpretation of information from large scale surveys of the classical Cepheid instability strip. We report here new results in a long-term program to determine the masses of Cepheids to an accuracy which can constrain evolutionary calculations. Orbits for galactic Cepheids have been derived and combined with orbital velocity amplitude measurements of hot companions from satellites (Hubble Space Telescope [HST] and IUE).

\section{Orbit}

The most recent system studied in this series is the long period ( $27 \mathrm{~d}$ ) Cepheid, $T$ Mon. New velocity data have been obtained and a new pulsation curve derived. The pulsation period has recently undergone a substantial change. While not quite a full orbit has been observed since the first velocities in 1905, both orbital maximum and minimum velocity have now been covered, and velocity observations within a few years will provide a complete orbit.

\section{Companion}

We have also measured the velocities of the companion from an HST GHRS spectrum and three IUE spectra. The velocities of the companion appear to be inconsistent with binary orbital motion from the preliminary orbit, and it is likely that the companion is itself a short period binary. The brightest companion is a chemically peculiar star, probably magnetic. The mass function from the preliminary orbit implies that the inclination of the long period system is close to 90 degrees. The long period of the system makes it a good candidate for resolution. Full results are given in Evans et al. (1999).

Acknowledgments. Financial Support was provided by NASA grants GO5868.02-91A to NRE and GO-4541.02 to KGC, the AXAF Science Center NASA Contract NAS8-39073, a grant from the Natural Sciences and Engineering Council, Canada to NRE, Hungarian OTKA grants T022946 and T029013 to LSz and F022249 to JV, and Hungarian Eötvös Fellowship and Magyary Postdoctoral Scholarship to JV.

\section{Reference}

Evans, N. R., Carpenter, K., Robinson, R., Massa, D., Wahlgren, G., Vinkó, J., \& Szabados, L. 1999, ApJ, 524, 379 